\title{
IMPACT OF ROTATIONAL SPEED OF COMPOSITE FRICTION PAIR ON ENERGY ACCUMULATION IN ACOUSTIC EMISSION SIGNAL FORMATION
}

\author{
Sergey Filonenko ${ }^{1}$, Aleksandr Kosmach ${ }^{2}$ \\ ${ }^{1}$ National Aviation University, 1 Kosmonavta Komarova Ave., Kiev 03680, Ukraine \\ ${ }^{2}$ Chernigiv State Technological University, Ukraine \\ E-mail:eduicao@nau.edu.ua; fils01@mail.ru (corresponding author)
}

Received 27 September 2013; accepted 20 November 2013

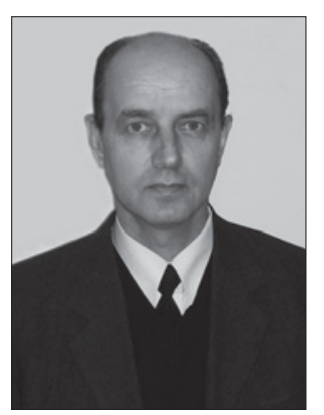

Sergey FILONENKO, Prof. Dr

Date and place of birth: 1954, Echmiadzin, Armenia.

Education: Kiev Polytechnic Institute.

Affiliation and functions: director of the Institute of Information Diagnostic

Systems at the National Aviation University since 2008.

Research interests: diagnostics of technological processes, automatic

diagnostic systems.

Publications: over 240 scientific papers.

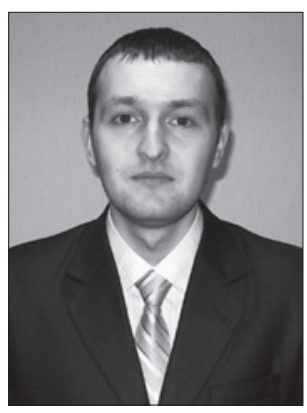

\begin{abstract}
Aleksandr KOSMACH, $\mathrm{PhD}$.
Date and place of birth: 1986, Chernigiv, Ukraine.

Education: National Aviation University.

Affiliation and functions: senior lecturer in the Department of Mechanical Engineering at Chernigiv State Technological University since 2013.

Research interests: diagnostics of technological processes, signal processing.

Publications: over 40 scientific papers.
\end{abstract}

\begin{abstract}
The paper considers the results of modelling acoustic emission signals that form under variations in the rotational speed of a friction pair made of a composite material. It is found that the energy accumulation in acoustic emission signals that form relative to time takes place in accordance with linear law under normal wear of a friction pair. If the rotational speed of a friction pair increases, the rates of accumulated average energy and accumulated total energy of the acoustic emission signals that form also grow. The resulting acoustic emission signals have both accumulated average energy and accumulated total energy. A comparison of these two types of energy is conducted. It is shown that the application of data averaging reduces the rate of the accumulated energy of the acoustic emission signals that form.
\end{abstract}

Keywords: acoustic emission, friction, composite material, rotation, signal, energy, accumulation. 


\section{Introduction}

Over the past few years, research of the frictional interaction between surfaces made of composite materials has been performed regularly in various countries of the world. The reason is that these materials have high tribological characteristics. This has led to wider application of composite materials in friction units in aircraft and the aerospace industry, as well as in transport, shipbuilding and other types of equipment operating under extreme conditions.

During the research of friction units made of composite materials, some traditional methods of determining the mechanical characteristics of these units are applied. Dynamic methods of researching friction units with traditional crystal structure of materials have been widely used recently. The results of research have shown that traditional methods of checking friction units are sensitive to the macro-processes in the surface layers of materials during their interaction. Thus, the information obtained according to these methods is the key factor in the development of methods of evaluating friction units, including methods of examining these units. At the same time, the availability of various types and brands of composite materials, the features of their structure, and the specifics of their destruction during friction and wear require a systematic approach to improve the reliability of inspection and examination of friction units made of composite materials. Obtaining such information is possible only when using methods that are sensitive to the micro-processes developing in the surface layers of the frictional contact of a friction unit. One method of non-destructive testing is the method of acoustic emission, which has been widely used in the technical examination of products lately. Recording acoustic emission data during frictional interaction between surface layers of materials provides an opportunity to receive a considerable amount of information about the kinetics of processes of friction and wear in progress. It should be noted that the flow and structure of acoustic emission data can change depending on variable conditions of these processes. It is necessary to add that the high sensitivity of the acoustic emission method and large volume of data recorded complicate its interpretation, so this method cannot be effectively implemented. First of all, it concerns the consequences of relationships between the parameters of processes in progress, or between parameters that characterise friction and wear processes and parameters of acoustic emission formation. As a result, some difficulties take place in the development of evaluation and diagnostic methods for friction units made of composite materials.

\section{Research methods}

Theoretical research related to the interpretation of acoustic emission data has a special meaning. This research is associated with the modelling and development of acoustic emission signals forming during friction and wear of the surface layers of composite materials. All these processes are therefore the basis for assessing the influence of various factors on acoustic emission formation, as well as for obtaining the regularities of its variable parameters that should be expected during experimental research.

Friction units of composite materials are studied in various ways and associated mainly with the selection of materials used to make interacting surfaces, selection of lubricants, optimisation of the modes of friction unit operations, and development of methods to check and evaluate friction units. Analysis of traditional mechanical characteristics (the friction moment, friction force, and friction coefficient) has shown that the use of composite materials often leads to a decrease in the friction coefficient and intensive wear of surface layers in frictional contact (Threrujirapapong et al. 2008; Terajima et al. 2009; Il-Young Kim et al. 2009; Nuruzzaman et al. 2010; Umanath et al. 2011; Bria et al. 2011). At the same time, on-going fractographic studies have revealed different mechanisms of the destruction of surface layers in frictional contact, including their fragile destruction. These types of destruction considerably decrease the tribological characteristics of friction units and indicate the initial phases of the irreversible catastrophic stage of wear. However, when using traditional methods of checking operating friction units, the observation of these stages gets complicated due to their low sensitivity and high inertness.

The acoustic emission method is used in studies of friction surfaces and wear of composite materials. The results of experimental research show that various factors can influence acoustic emissions, among them strong interaction between surfaces (pressure on a friction unit), the contact surface rate of change (rotational speed of a friction pair), lubricating layers, the geometric parameters of surface micro-elements (type and form of the roughness of surfaces in contact), the physical and mechanical properties of surface micro-elements, etc. It is necessary to emphasise the complexity of the acoustic emissions recorded during the operation of friction units made of materials with traditional structure and composite materials (Hase et al. 2009; Fan et al. 2010; Wang, Wood 2007; Polok-Rubiniec et al. 2009; Tillmann et al. 2009; Pakula 2013). Interpretation of acoustic emission formation has considerable difficulties with the further search for regularities in the variation of acoustic emission parameters, especially at the stage of normal wear. The complexity of interpreting acoustic emissions is 
also related to the complexity of the theoretical research of acoustic emission signals that form during friction between surfaces made of composite materials.

Some references used in this paper consider a mathematical model and modelling of resulting acoustic emission signals formed during friction between surfaces of traditional crystal structure taking into account the contact surface rate of change, influence of constant axial loading, contact surface area of interaction, size of material under plastic deformation, and predominant destruction of secondary structures of type I and II (Filonenko et al. 2008). This research allows determining the main regularities of resulting acoustic emission signal parameters when the variation of influencing factors takes place during normal wear and during the transition from normal stage of wear to irreversible catastrophic stage of wear. This suggests that the theoretical results perfectly comply with the experimental results (Filonenko et al. 2008, 2009).

The concept of FBM (fibre bundle models) can be a concept for the creation of models and research of processes of friction between surfaces made of composite materials (Shcherbakov 2002). However, most of the research this concept is used in is related to models and modelling of the destruction of elements made of composite materials under tension and shear force influence (Shcherbakov 2002; Kun et al. 2007; Raischel et al. 2005). Research of the destruction of composite material under tension with analysis of the energy release rate of acoustic emission events has also been conducted (Shcherbakov 2002). It has been proven that when the time of the complete destruction of composite materials draws nearer, the energy release rate of acoustic emissions increases. However, at the moment of the complete destruction of a composite material, the continuality of the function can be broken, i.e. the function that describes the changes in the accumulated energy of acoustic emission events. This situation does not allow us to get an analytical expression for the model signal of acoustic emissions.

A model based on the concept of fibre bundle models and the results of the modelling of acoustic emission signals forming during the destruction of composite materials under shear load were considered (Filonenko et al. 2012). Taking into account some features of the kinetics of composite material destruction, the expression that describes this process and the expression for the acoustic emission signal that forms were obtained. It was confirmed that composite material destruction with the specified quantity of elements leads to the formation of an acoustic emission signal as a visual impulse. In this case, the basic regularities of variation in the amplitude and energy parameters of acoustic emission signals affected by various factors can be determined. These factors are the following: the rate of composite material loading, the physical and mechanical characteristics of the composite material, and the size of the elements. The expression describing an acoustic emission impulse signal forming during the period of composite material destruction under the influence of shear load is as follows:

$$
\begin{aligned}
U(t)= & U_{0} \mathrm{v}_{0}\left[\alpha t(1-\alpha t)(1-g \sqrt{\alpha t})-\alpha t_{0}\left(1-\alpha t_{0}\right)\left(1-g \sqrt{\alpha t_{0}}\right)\right] \times \\
& \times e^{r\left[\alpha t(1-\alpha t)(1-g \sqrt{\alpha t})-\alpha t_{0}\left(1-\alpha t_{0}\right)\left(1-g \sqrt{\alpha t_{0}}\right)\right]} \times \\
& \quad-\mathrm{v}_{0} \int^{t} e^{r\left[\alpha t(1-\alpha t)(1-g \sqrt{\alpha t})-\alpha t_{0}\left(1-\alpha t_{0}\right)\left(1-g \sqrt{\alpha t_{0}}\right)\right]} d t
\end{aligned},
$$

where $N_{0}$ is the initial quantity of composite material elements facing destruction; $\mathrm{U}_{0}$ and $r$ are constants that depend on the physical and mechanical characteristics of the composite material; $\alpha$ is the rate of loading of the composite material; $t$ is the time, $t_{0}$ is the time of the beginning of element destruction; $g$ is the coefficient that indicates the geometrical sizes of elements; $U_{0}=N_{0} \beta \delta_{S}$ is maximum possible displacement at the instant of the destruction of elements; $\beta$ is the factor of proportionality; and $\delta_{S}$ is a parameter having a numerical value determined by a form of a single disturbance impulse at destruction of one element and calculated as a time unit.

Expression 1, similar to the case of the destruction of secondary structures of type I and II, which is mentioned above, can be used to analyse a resulting signal forming during friction between surfaces made of composite material. In this case, it is possible to consider some small (elementary) platform $S_{T}$ of contact interaction in the area $S$ overlapping the surfaces of a friction pair, within which there is a loss of a specified quantity of elements $N_{0}$ that have certain physical and mechanical characteristics and geometrical dimensions (Fig. 1). Thus, the rotational speed that provides a time-shift position of a contact interaction platform and the axial loading applied to a friction pair will determine the threshold tension at which the destruction of composite material begins. Under these conditions, it is possible to simulate the energy parameters of the resulting acoustic emission signals forming during friction between surfaces made of composite materials, taking into account the influence of various factors, one of which can be the rotational speed of the friction pair.

Given the conditions and approaches that are described above and are used to characterise the formation of an acoustic emission signal under friction and wear of frictional contact surfaces made of traditional materials, the resulting acoustic emission signal forming during friction between surfaces made of composite materials can be shown in the following form:

$$
U_{p}(t)=\sum_{j} U_{j}\left(t-t_{j}\right),
$$

where $j$ is the serial number of the contact interaction area $j$ $(j=0,1,2, \ldots, \mathrm{m}) ; U_{j}\left(t_{j}\right)$ is the impulse acoustic emission signal forming in the contact interaction area $j$ according to 
expression $1 ; t_{j}$ is the time that $j$ acoustic emission signal appears; and $m$ is the number of acoustic emission impulse signals during operating time $T$ of a friction unit.

According to expression 2, the resulting acoustic emission signal is given as a sum of impulse signals forming at specified consistent time points $t_{j}$ during stable destruction of elementary platforms, including a specified quantity of elementary contact interactions $N_{0}$. The change in elementary contact surfaces is determined by the rotational speed of the friction unit. The time point $t_{j}$ when an i acoustic emission impulse signal appears can be given as a fixed component and as a random component:

$$
t_{j}=j \Delta t_{j} \pm \delta
$$

where $\Delta t_{j}$ is the time interval between the beginning of the appearance of a subsequent acoustic emission impulse signal and a preceding one, and $\delta$ is a random component of the time when each subsequent acoustic emission signal appears.

The use of the random component $\delta$ in expression 3 is caused by the dispersion that pertains to the properties of composite materials on a friction surface, as well as by the possible unstable position of platform $S_{T}$ of contact interaction (the platform facing destruction) in the total area $S$ overlapping frictional contact surfaces (Fig. 1). It should be noted that the conditions described above are maintained despite the use of different patterns of contact interaction.

\section{Results of theoretical research}

Modelling the energy characteristics of the resulting acoustic emission signals can be conducted according to equation (2) using equation (3) when the rotational speed of a friction unit made of composite materials varies. All the parameters in equations (1), (2) and (3) will be given dimensionless quantities when modelling. The process of modelling will be carried out in relative units taking some certain conditions into consideration. The quantity of $N_{0}$ elements on each subsequent platform $S_{T}$ (Fig. 1) is a constant. It is necessary to add that these $N_{0}$ elements have an identical size.

The values of parameters $\tilde{v}_{0}, \tilde{r}$ and $\tilde{g}$ in expression (1) are assumed to be as follows: $\tilde{\mathrm{v}}_{0}=10^{6} ; \tilde{r}=10^{4}$; $\tilde{g}=0.1$. The value $\tilde{\alpha}$ is changed in the range of 200 to 600 with the step of an increment $\Delta \tilde{\alpha}$ equal to 100 . The threshold tension $\tilde{\sigma}_{0}$ of the destruction of composite material elements is assumed to be constant. Its value can be taken as $\tilde{\sigma}_{0}=0.101941909$. Under constant $\tilde{\sigma}_{0}$, start times for the destruction of composite material elements are determined as relative units for the specified values $\tilde{\alpha}$ according to the methods that have been developed (Filonenko et al. 2012). It is accepted that if $\tilde{\alpha}$ equals 200 the value of start time $\tilde{t}_{0}$ for the destruction of composite material elements can be taken as $\tilde{t}_{0_{200}}=$

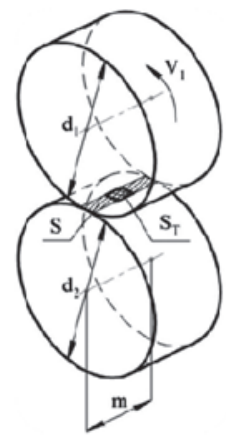

a

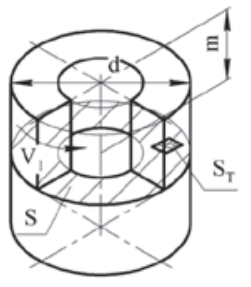

b
Fig. 1. Kinematic scheme of friction units in the form of rings with cylindrical interaction $(a)$ and disks with front interaction (b): $V$ - rotational speed of a friction unit, $S$ - area overlapping the surfaces of the friction pair; $S_{T}-$ contact platform in the area overlapping the surfaces of the friction pair; $m$ and $d-$ sizes of composite material elements on the contact surface

0.0006. According to the calculations conducted, given the accepted speeds of loading and under the assumption that $\tilde{\sigma}_{0}=0.101941909$, the values of start times for the destruction of composite material elements are taken as follows: $\tilde{\alpha}=300-\tilde{t}_{0_{300}}=0.0004 ; \tilde{\alpha}=400-\tilde{t}_{\underline{Q}_{400}}=$ 0.0003; $\tilde{\alpha}=500-\tilde{t}_{0_{500}}=0.00024 ; \tilde{\alpha}=600-t_{0_{600}}=$ 0.0002 .

The value of the time interval $\Delta \tilde{t}_{j}$ between the previous and the subsequent appearance of acoustic emission signals is accepted as $\Delta \tilde{t}_{j}=1.1 \times 10^{-6}$ under the condition that $\tilde{\alpha}=200$. This time is chosen on the basis of the duration of an acoustic emission signal forming under the condition that $\tilde{\alpha}=200$. According to expression (1), this duration is determined when calculating the amplitude of a signal relative to time. Pertaining to the condition that $\tilde{\alpha}=200$, the value $\tilde{\delta}$ varies randomly in the range of values $\tilde{\delta}=0 . .5 .0 \times 10^{-7}$. If the value $\tilde{\alpha}$ increases, the duration of the acoustic emission impulse signal that forms will decrease, as proved by the results of research that was performed recently (Filonenko et al. 2012). Accordingly, the values of $\Delta \tilde{t}_{j}$ and $\tilde{\delta}$ are changed proportionally to the condition that $\tilde{\alpha}$ equals 200 for other $\tilde{\alpha}$ values.

The modelling is carried out in several stages. The first stage includes calculations of the energy of a resulting acoustic emission signal, in particular its energy time variations, according to expression (2) and pertaining to the conditions described above. The second stage includes the calculation of the accumulated energy of acoustic emission signals and their time characteristics according to the results obtained for each accepted rotational speed of a friction unit. The third stage is to determine the influence of the rotational speed of a friction unit and the regularities of rotational speed variations on the rate of the accumulated energy of the acoustic emission signals that form. 
Figure 2 shows some modelling results as regularities of the variable energy $\tilde{E}$ and accumulated energy $\tilde{E}_{N}$ of resulting acoustic emission signals for the values $\sqrt{K}=300$ and $\tilde{\alpha}=600$.

Analysis of the modelling results showed that at constant values of the parameters that characterise the physical and mechanical properties of composite materials $\left(\tilde{v}_{0}, \tilde{r}\right)$ and the size of composite material components $(\tilde{g})$, the values of average energy $\tilde{E}$ of the resulting acoustic emission signals and the value of acoustic emission signal dispersion increase simultaneously with $\tilde{\alpha}$ values (increase in the rotational speed of a friction unit).

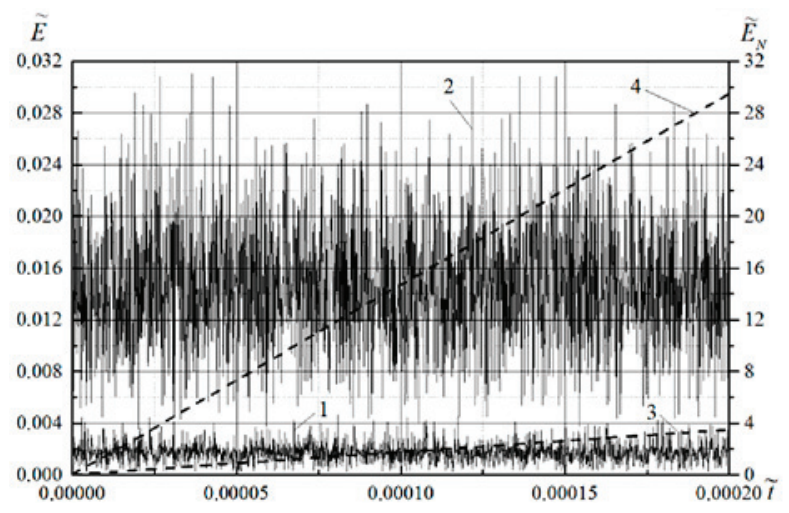

Fig. 2. Diagrams of variable energy $\tilde{E}(1,2)$ and accumulated energy $\tilde{E}_{N}(3,4)$ of resulting acoustic emission signals and their time characteristics according to expression (2), where $\tilde{\alpha}$ equals 300 and $\tilde{\alpha}$ equals 600 . Modelling parameters according to expression (1) are the following: $\tilde{\mathrm{v}}_{0}=10^{6}$; $\tilde{r}=10^{4} ; \tilde{g}=0,1 ; \quad \tilde{\sigma}_{0}=0,101941909$

Figure 3 shows the variations in the accumulated energy $\tilde{E}_{N}$ of a resulting acoustic emission signal and the rate of energy accumulation when the rotational speed of friction unit $\tilde{\alpha}$ varies. The rate of the variable accumulated energy $\tilde{E}^{\prime}$ of the acoustic emission signal that forms are determined by the dependence of the accumulated energy of an acoustic emission signal on the time interval of accumulation.

As the analysis of the data obtained shows, the value $\tilde{E}^{\prime}$ is equal to 4867.68 when $\tilde{\alpha}$ is equal to 200 . When value $\tilde{\alpha}$ is increased 1.5 times (when $\tilde{\alpha}$ is equal to 300 ), the $\tilde{E}^{\prime}$ values increase 3.59 times. This kind of increase can be observed simultaneously with the further increase in value $\tilde{\alpha}$. Under the condition that $\tilde{\alpha}$ is equal to 400 , the $\tilde{E}^{\prime}$ values increase 8.84 times. When value $\tilde{\alpha}$ is increased 2.5 times (when $\tilde{\alpha}$ is equal to 500 ) the $\tilde{E}^{\prime}$ values increase 17.38 times. When value $\tilde{\alpha}$ is increased 3 times (when $\tilde{\alpha}$ is equal to 600 ) the $\tilde{E}^{\prime}$ values increase 30.28 times.

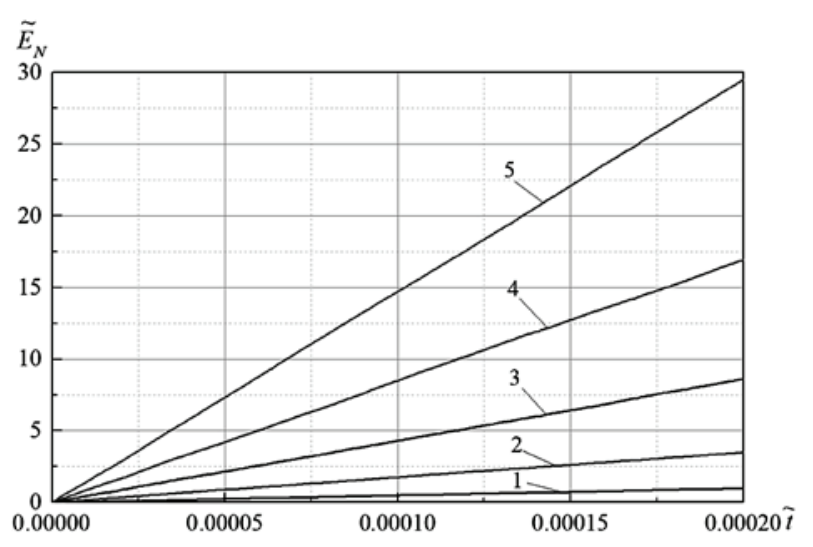

a

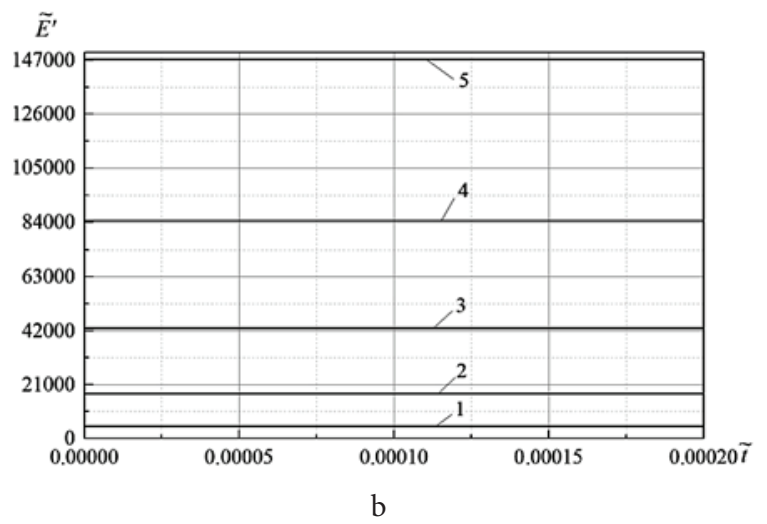

Fig. 3. Diagrams of variable accumulated energy $n_{C} \quad$ (a) and rate of accumulated energy $\tilde{E}^{\prime}(b)$ of a resulting acoustic emission signal and their time characteristics according to expression (2) and pertaining to the accepted values of $\tilde{\alpha}$, which are the following: $1-\tilde{\alpha}=200,2-\tilde{\alpha}=300,3-$ $\tilde{\alpha}=400,4-\tilde{\alpha}=500,5-\tilde{\alpha}=600$. Modelling parameters according to expression (1) are as follows: $\tilde{\mathrm{v}}_{0}=10^{6} ; \tilde{r}=10^{4}$; $\tilde{g}=0.1 ; \quad \tilde{\sigma}_{0}=0.101941909$

Figure 4 shows variations in the rate of accumulated energy $\tilde{E}^{\prime}$ of a resulting acoustic emission signal and the percentage of increase in energy accumulation under variation of the rotational speed of friction unit $\tilde{\alpha}$.

Figure 5 shows that the accumulated average energy and accumulated total energy of resulting acoustic emission signals grow continuously and linearly. This increase in acoustic emission signal parameters is characterised by the rate of energy accumulation, which was determined by the dependence of the accumulated energy of an acoustic emission signal on the time interval of accumulation. 

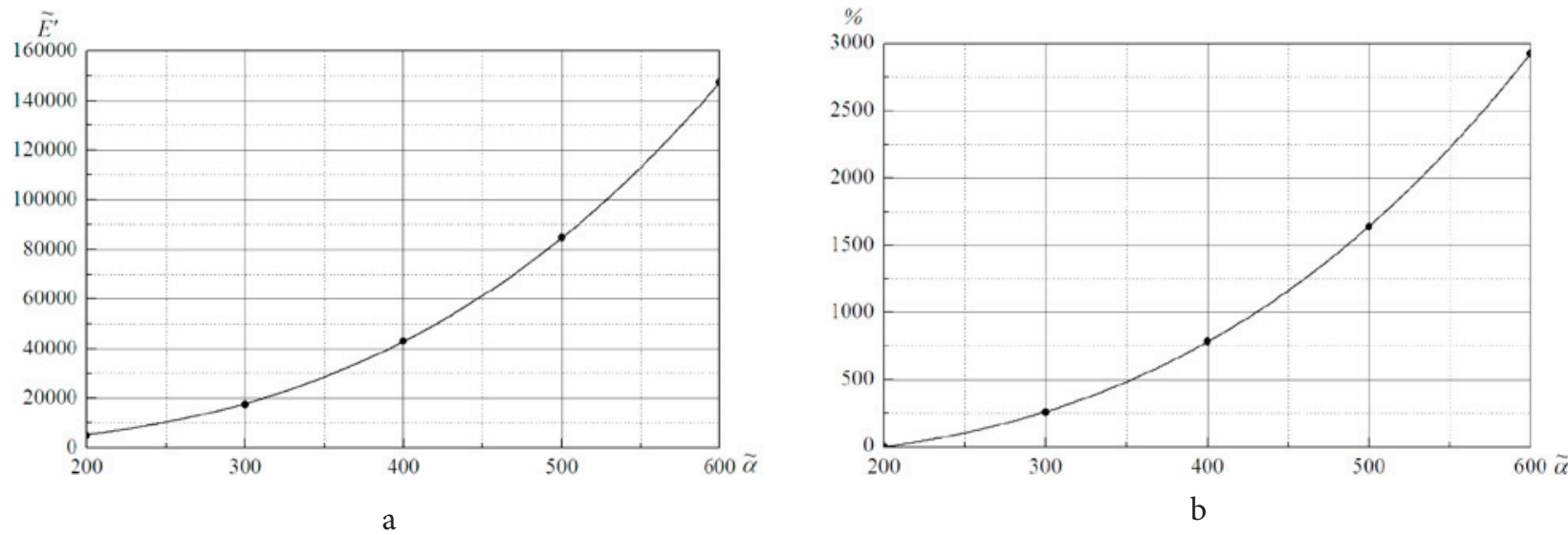

Fig. 4. Diagrams of the variation in the rate of accumulated energy $\tilde{E}^{\prime}(a)$ and the percentage of increase in the rate of accumulated energy $(b)$ of a resulting acoustic emission signal depending on the value $\tilde{\alpha}$ when $\tilde{\alpha}$ is equal to 200

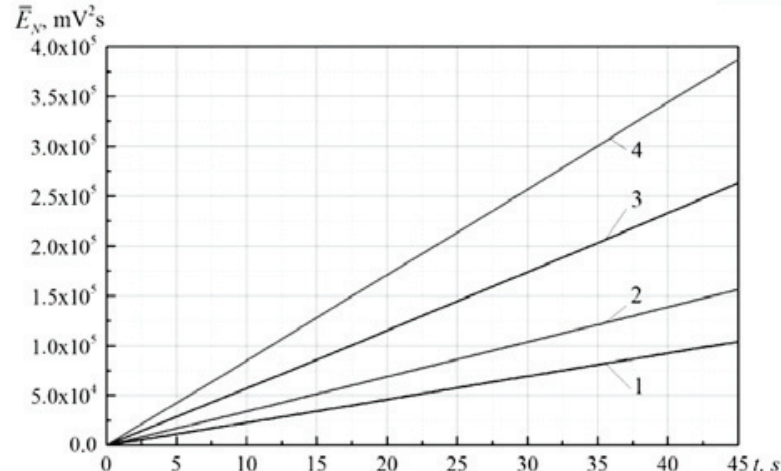

a

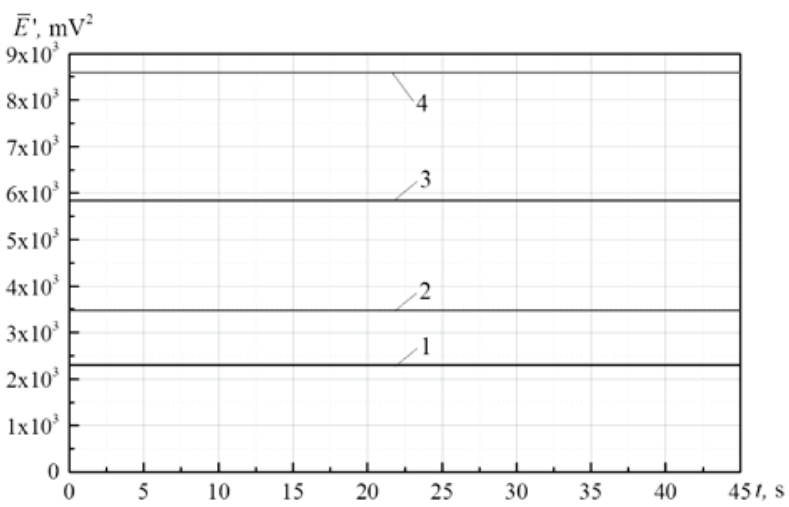

b
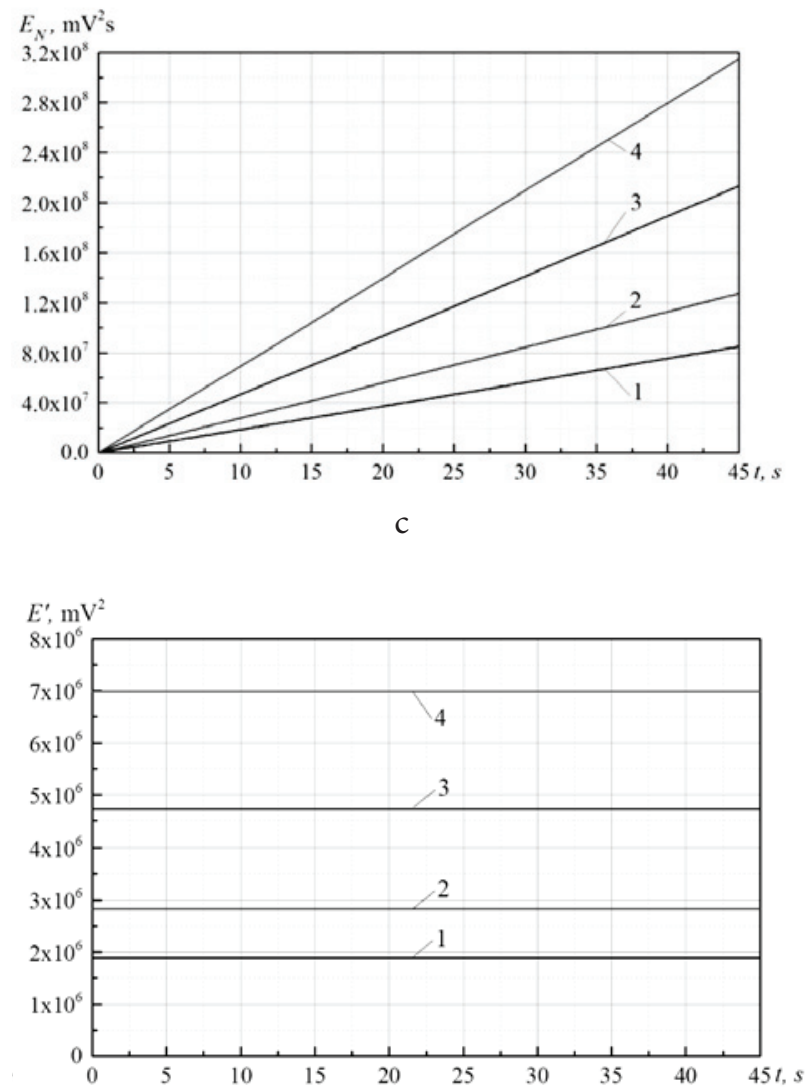

d

Fig. 5. Accumulated average energy $\bar{E}_{N}(a)$ and accumulated total energy $E_{N}(c)$ of acoustic emission signals; the rate of accumulated energy $\bar{E}^{\prime}$ and the rate of accumulated energy $E^{\prime}(b, d)$, which were recorded at different rotational speeds $v$ at a normal stage of wear of a friction pair with a coating made of composite material. The rotational speeds of friction pair $v$ are the following: $1-500 \mathrm{~min}^{-1}, 2-600 \mathrm{~min}^{-1}, 3-700 \mathrm{~min}^{-1}, 4-800 \mathrm{~min}^{-1}$. The axial loading on a friction unit is taken as $P=450 \mathrm{~N}$ 
Figure 6 shows diagrams of accumulated average energy $\bar{E}_{N}$ and accumulated total energy $E_{N}$ of a resulting acoustic emission signal and the rates of accumulated energies $\bar{E}^{\prime}$ and $E^{\prime}$ when the rotational speed of friction unit $v$ varies.
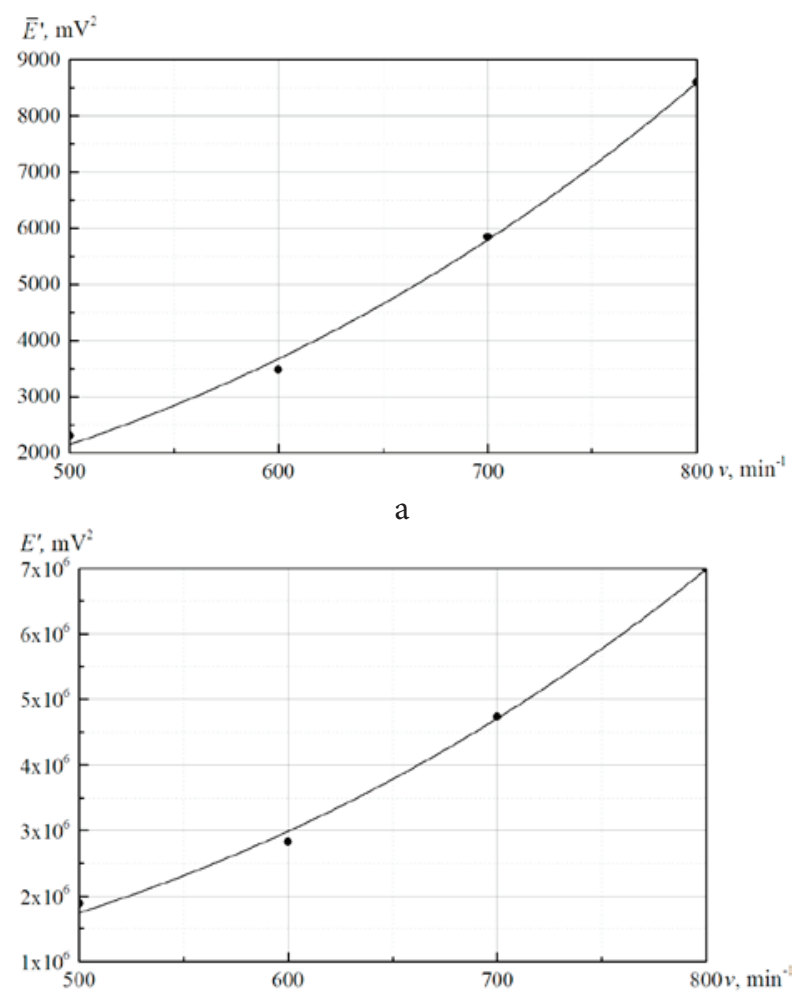

$\mathrm{b}$

Fig. 6. Diagrams of accumulated average energy $\bar{E}^{\prime}$ (a) and accumulated total energy $E^{\prime}(b)$ of a resulting acoustic emission signal depending on the rotational speed of a friction pair $v$

As the analysis of the data obtained shows, if speed $v$ is equal to $500 \mathrm{~min}^{-1}$, the values of the rates of accumulated average energy $\bar{E}^{\prime}$ and accumulated total energy $E^{\prime}$ of acoustic emission signals that form will be taken as $\bar{E}^{\prime}=2306.57 m V^{2}$ and $E^{\prime}=1.88 \times 10^{6} m V^{2}$. When value $v$ is increased 1.2 times (to $600 \mathrm{~min}^{-1}$ ) the values $\bar{E}^{\prime}$ and $E^{\prime}$ increase, respectively, 1.505 and 1.498 times. This kind of increase can be observed simultaneously with the further increase in the rotational speed of a friction pair. So, if speed $v$ is equal to $700 \mathrm{~min}^{-1}$, the values $\bar{E}^{\prime}$ and $E^{\prime}$ will increase, respectively, 2.534 and 2.51 times. When $v$ is increased 1.6 times (to $800 \mathrm{~min}^{-1}$ ), the values $\bar{E}^{\prime}$ and $E^{\prime}$ will increase, respectively, 3.725 and 3.71 times.

Regularities in the variations of accumulated average energy and accumulated total energy of a resulting acoustic emission signal are characterised by non-linear specific functions when the rotational speed of a friction pair made of composite materials increases.
According to the analysis of the data obtained, the coefficients of approximating expression (4) pertaining to the regularities analysed are taken as follows: $a=$ $2.28 \times 10^{-5}, b=2.9542$ (the rate of accumulated average energy $\bar{E}^{\prime}$ of an acoustic emission signal); $a=0.01847$, $b=2.9546$ (the rate of accumulated total energy $E^{\prime}$ of an acoustic emission signal). The determining coefficient of the regularities received is taken as $\mathrm{R}^{2}=0.995$.

\section{Conclusions}

The results of acoustic emission modelling have shown that there is a continuous linear accumulation of the energy of an acoustic emission signal resulting from friction between surfaces made of composite materials. The accumulated energy of an acoustic emission signal can be characterised by the accumulation rate.

The increase in the rate of accumulated energy of acoustic emission signals and the increase in the rotational speed of a friction pair made of composite materials have been theoretically considered and experimentally confirmed.

The statistical analysis of the data obtained and approximation of regularities in the rate variations pertaining to accumulated average energy and accumulated total energy of the resulting acoustic emission signals have been carried out. It was determined that the increase in the average energy and total energy of an acoustic emission signal can be considered identical in the investigated range of rotational speeds of a friction pair.

The results obtained during research lead us to conclude that if averaging is used, the rate of the accumulated energy of a resulting acoustic emission signal will be much less than the same rate without averaging. If one takes into consideration the inspection of friction units made of composite materials, it is clear that the accumulated average energy of acoustic emission signals ought to be used as an informative parameter of an acoustic emission signal in order to record smaller amounts of data. With this in mind, it is necessary to emphasise that the experimental results have perfectly complied with the results of theoretical research.

\section{References}

Bria, V.; Dima, D.; Andrei, G., et al. 2011. Tribological and wear properties of multi-layered materials, Tribology in Industry 33(3): 104-9 [online], [cited 10 March 2013]. Available from Internet: http://www.tribology.fink.rs/journals/ 2011/2011-3/2.pdf

Fan, Y.; Gu, F.; Ball, A. 2010. Modeling acoustic emissions generated by sliding friction, Wear 268(5-6): 811-815. http://dx.doi.org/10.1016/j.wear.2009.12.010

Filonenko, S.; Kalita, V.; Kosmach, A. 2012. Destruction of composite material by shear load and formation of acoustic emission, Aviation 16(1): 5-13.

http://dx.doi.org/10.3846/16487788.2012.679831 
Filonenko, S.; Stadnichenko, V.; Stahova, A. 2008. Modelling of acoustic emission signals at friction of materials' surface layers, Aviation 12(3): 87-94. http://dx.doi.org/10.3846/1648-7788.2008.12.87-94

Filonenko, S.; Stadnichenko, V.; Stahova, A. 2009. Definition of contact stress in friction units on the basis of the acoustic emission method, Aviation 13(3): 72-77. http://dx.doi.org/10.3846/1648-7788.2009.13.72-77

Hase, A.; Wada, M.; Mishina, H. 2009. Acoustic emission in elementary processes of friction and wear: in-situ observation of friction surface and AT signals, Journal of Advanced Mechanical Design, Items and Manufacturing 3(4): 333-344.

Il-Young, K.; Jung-Hee, L.; Gyu-Sun, L., et al. 2009. Friction and wear characteristics of the carbon nanotube-aluminum composites with different manufacturing conditions, Wear 267(1-4): 593-598.

Kun, F.; Raischel, F.; Hidalgo, R. C., et al. 2007. Extensions of fibre bundle models, Modelling Critical and Catastrophic Phenomena in Geoscience. Lecture Notes in Physics 705(1): 57-92.

Nuruzzaman, D. M.; Nakajima, A.; Mawatari, T., et al. 2010. Experimental study on durability and tribological properties of HVOF sprayed WC cermet coatings, Journal of Advanced Research in Mechanical Engineering 1(4): 203209 [online], [cited 10 March 2013]. Available from Internet: http://www.hypersciences.org/ JARME/Iss.4-2010/ JARME-4-4-2010.pdf

Pakula, D. 2011. Structure and properties of multicomponent coatings deposited onto sialon tool ceramics, Archives of Materials Science and Engineering 52(1): 54-60.

Polok-Rubiniec, M.; Dobrzanski, L. A.; Adamiak, M. 2009. Comparison of the PVD coatings, Archives of Materials Science and Engineering 38(2): 118-125.

Raischel, F.; Kun, F.; Herrmann, H. J. 2005. Simple beam model for the shear failure of interfaces, Physical Review E. 72(1): $1-11$.
Shcherbakov, R. 2002. On Modeling of Geophysical Problems: Doctoral Dissertation [online], [cited 10 March 2013]. Cornell University. 209 p. Available from Internet: http:// www.msi.umn.edu/ lilli/ Russian-Bobby-thesis.pdf.

Terajima, T.; Takeuchi, F.; Nakata, K., et al. 2009. Tribological properties of $\mathrm{WC} / 12 \mathrm{Co}$ cermet-Fe-Based metallic glass spray coating, Transactions of JWRI 38(2): 75-79 [online], [cited 10 March 2013]. Available from Internet: http://www. jwri.osaka-u.ac.jp/ publication/trans-jwri/pdf/381-13.pdf

Threrujirapapong, T.; Kondoh, K.; Umeda, J., et al. 2008. Friction and wear behavior of titanium matrix composite reinforced with carbon nanotubes under dry conditions, Transactions of JWRI 37(2): 51-56 [online], [cited 10 March 2013]. Available from Internet: http://www.jwri.osaka-u. ac.jp/ publication/trans-jwri/pdf/372-07.pdf

Tillmann, W.; Vogli, E.; Gathen, M., et al. 2009. Development of wear resistant pressing moulds for the production of diamond composites, Journal of Materials Processing Technology 209(9): 4268-4273. http://dx.doi.org/10.1016/j.jmatprotec.2008.11.005

Umanath, K.; Selvamani, S. T.; Palanikumar, K. 2011. Friction and wear behavior of Al6061 alloy ( $\mathrm{SiCP}+\mathrm{Al} 2 \mathrm{O} 3 \mathrm{P})$ hybrid composites, International Journal of Engineering Science and Technology 3(7): 5441-5451.

Wang, L.; Wood, R. J. K. 2007. The influence of contact conditions on surface reaction layers formed between steel surfaces lubricated by an aviation oil, Tribology International 40(10): 1655-1666. http://dx.doi.org/10.1016/j.triboint.2007.02.014 